\title{
An adult male with abdominal pain and skin rash
}

\author{
Jay Patel MD, Imran Umer MD, Anand Reddy MD, \\ Yasir Ahmed MD, Craig Spellman DO, PhD
}

\begin{abstract}
Henoch-Schönlein purpura is generally a disease of children and uncommon in adults, especially after the age of 40 . It is characterized by leukocytoclastic vasculitis skin rash, arthralgia, and gastrointestinal symptoms. In adults, glomerulonephritis may occur and the long-term prognosis is poor. We present the case of a 65-year-old man with complaints of abdomen pain and skin rash who subsequently developed renal failure. He was diagnosed with adult onset Henoch-Schönlein purpura and survived after treatment with glucocorticoids and hemodialysis.
\end{abstract}

Key words: Henoch-Schönlein purpura, HSP, IgA vasculitis, leukocytoclastic vasculitis, acute renal failure, skin rash

\section{INTRODUCTION}

Henoch-Schönlein purpura (HSP) involves the small blood vessels and presents with a purpuric skin rash, arthralgia, and gastrointestinal symptoms. HSP is usually an autoimmune disease in children and occurs after viral infections. HSP is rare in adults, is more severe, and carries a poor prognosis compared to children. 'Glomerulonephritis is seen in approximately $50 \%$ of patients and is associated with a poor long-term prognosis. ${ }^{2}$ Mortality in adults with HSP is usually due to gastrointestinal complications, including ischemic colitis, perforations, and bleeding. ${ }^{3}$ Here we report a case of adult onset HSP who developed renal failure and survived after a prolonged hospital course requiring supportive care, glucocorticoid therapy, and temporary hemodialysis.

Corresponding author: Yasir Ahmed MD

Contact Information: Yasir.ahmed@ttuhsc.edu

DOI: 10.12746/swrccc2015.0309.118

\section{Case Presenttion}

A 65-year-old Caucasian man with history of hypertension presented with abdominal pain and a skin rash with swelling involving both lower extremities for one week. Prior to admission, he visited an urgent care clinic and received an intramuscular injection of methylprednisolone. He developed nausea and vomiting with increased abdominal pain and was admitted in our hospital. He also complained of diffuse arthralgia but denied fever, chills, diarrhea, recent travel, and contact with ill persons. He denied any prior history of skin rashes or lesions. Vital signs included temperature $98^{\circ} \mathrm{F}$, blood pressure $130 / 82 \mathrm{mmHg}$, pulse rate 92 beats/minute, respiratory rate 20 breaths/minute, and oxygen saturation $95 \%$ on room air. Physical examination was significant for diffuse abdominal tenderness and petechial skin lesions involving the legs and feet. (Figure-1) 


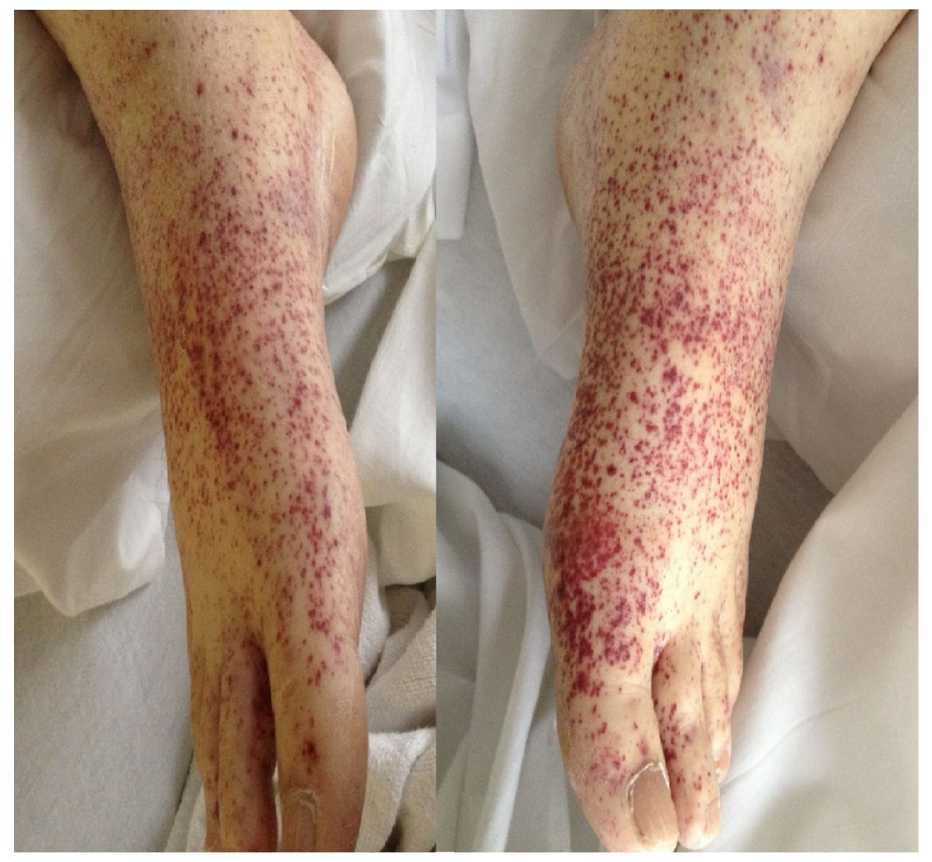

Figure 1. Right and left foot and ankle showing petechial and purpuric skin rash

The initial laboratory studies revealed white blood cell count $17 \times 10^{3} / \mu \mathrm{L}$ (polymorph $91 \%$, lymphocytes $6 \%$ ), hemoglobin $14 \mathrm{gm} / \mathrm{dL}$, platelets $41 \times 10^{3} / \mu \mathrm{L}$, erythrocyte sedimentation rate $8 \mathrm{~mm} / \mathrm{hr}$. Liver function tests and serum electrolytes were normal except for creatinine of $1.6 \mathrm{mg} / \mathrm{dL}$. Urine studies showed protein $100 \mathrm{mg} / \mathrm{dL}$ and 10 red blood cells per high power field. Repeat urine protein studies showed proteinuria at $2014 \mathrm{mg}$ per $24 \mathrm{hrs}$. A computed tomography scan of the abdomen reported nonspecific thickening of the large intestine and consistent with early ischemic colitis. Empiric antibiotic therapy was begun and an infectious disease work up was done for HIV, hepatitis $B$ and $C$, and bacterial infections. By hospital day three, the petechial rash extended up to the abdomen, buttocks, and hands, and anasarca was evident. During the next five days, the patient became anuric and a transaminitis developed with increased aspartate aminotransferase levels (106 IU/L) and alanine aminotransferase levels (91 IU/L). His serum creatinine increased to $5.1 \mathrm{mg} / \mathrm{dl}$, and the BUN increased to $116 \mathrm{mg} / \mathrm{dl}$. All infectious disease work ups were reported to be negative so antibiotics were stopped on hospital day eight. Serologic studies for vasculitis of various etiologies (ANCAs, ANA, cryoglobulins, etc.) were negative. Based on the skin lesions, the abdominal pain and renal failure, the clinical diagnosis of HSP was made. Subsequently, skin and renal biopsies were performed and leukocytoclastic vasculitis and $\lg A$ nephropathy with chronic inflammatory changes was reported, respectively (Figure 2a, 2b, 2c). Methylprednisolone $500 \mathrm{mg}$ intravenous twice a day was administered on hospital days $5-8$, and the patient was then switched to oral prednisone $90 \mathrm{mg}$ per day. Hemodialysis was started on hospital day eight for acute renal failure and anuria. The patient slowly improved with resolution of the arthralgia, anasarca, abdominal pain and skin lesions. Dialysis was discontinued after three weeks, but oral prednisone was continued and weaned off after three months. At six weeks follow up visit as an outpatient, the patient remained symptom free with return of renal function to base line.

\section{Discussion}

HSP is typically a disease of children and is very uncommon in adults. ${ }^{4,5}$ The incidence in adults varies from approximately 3 to 14 cases per million, depending upon the particular study and patient ethnicity. ${ }^{6}$ It is an immune-mediated systemic leukocytoclastic vasculitis characterized by vascular and/or mesangial $\lg \mathrm{A}$ deposition.

The etiology of HSP remains unknown; it may be triggered by a variety of antigenic stimuli including infections, drugs, toxins, systemic diseases and cancer. $^{7,8}$ In the present case infectious gastroenteritis was possibly a triggering factor. Episodes of macroscopic hematuria are frequently associated with acute renal failure and HSP-associated IgA nephropathy leads to severe renal failure $(\mathrm{CrCl}<30 \mathrm{ml} / \mathrm{min})$ in $13 \%$ and end stage renal disease in up to $11 \%$ of adult patients on long term follow-up. Proteinuria, hypertension, and initial kidney impairment portend an unfavorable renal prognosis with the degree of pro- 


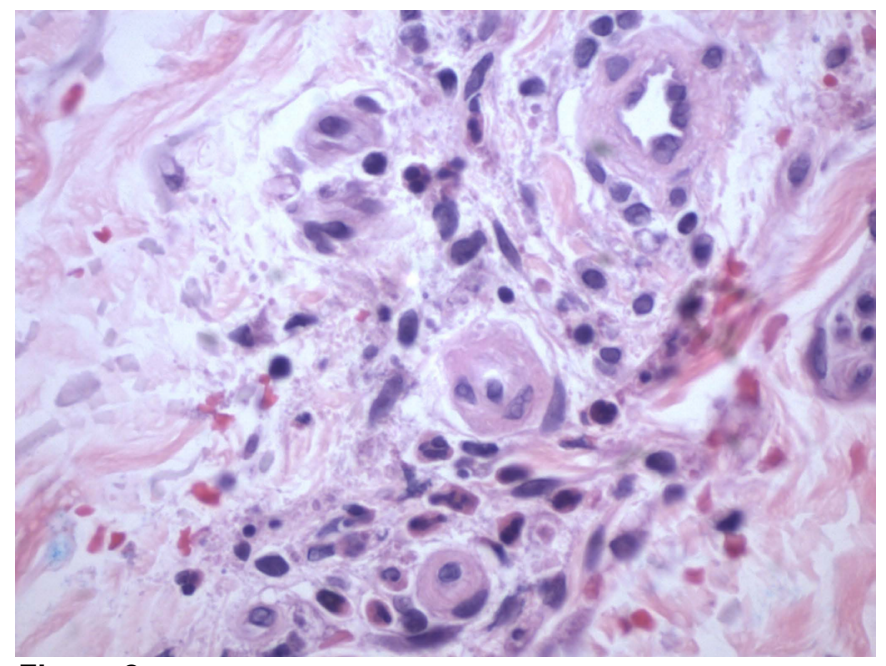

Figure 2a

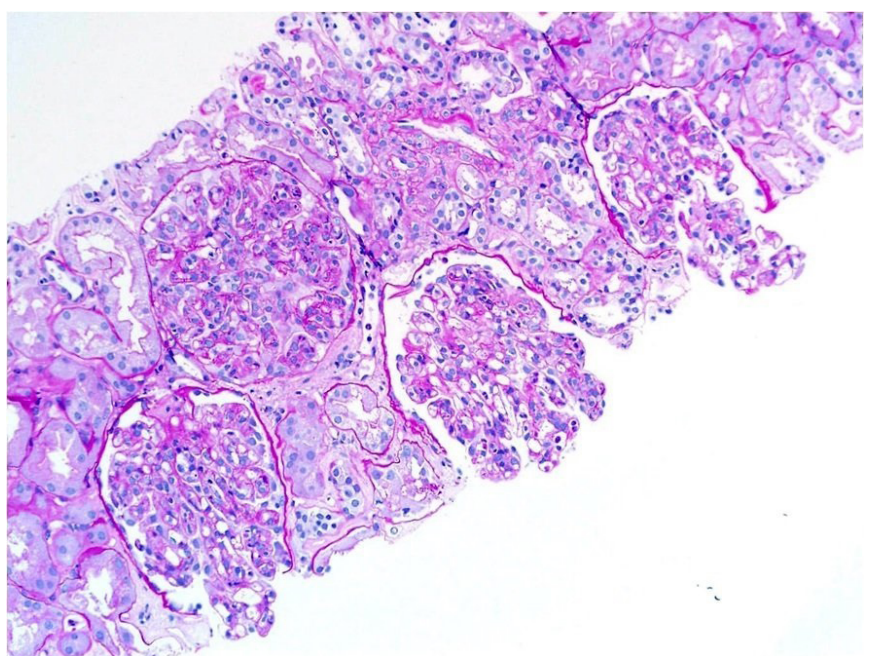

Figure 2b

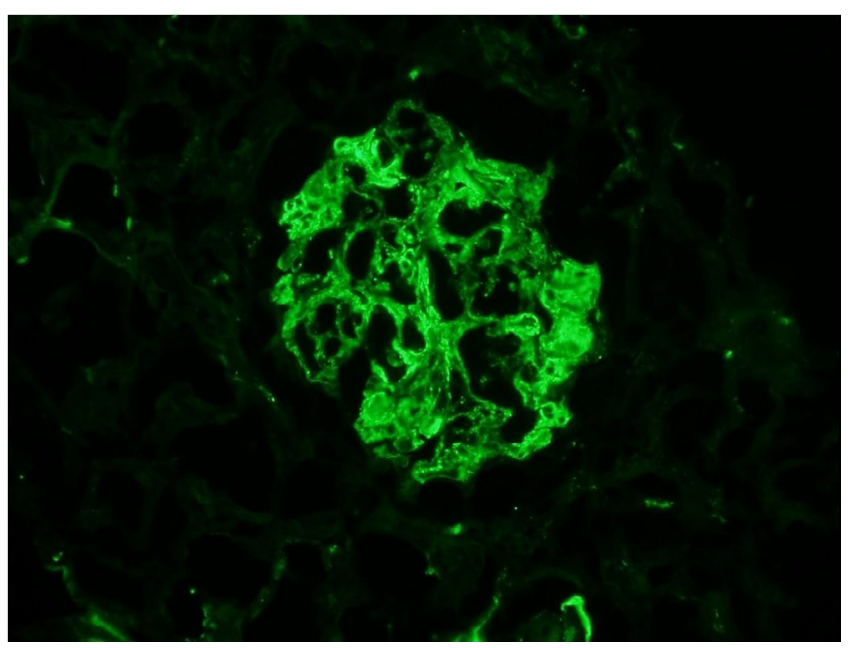

Figure 2c
Figure 2 a. skin biopsy, showing leukocytoclastic vasculitis; b. the glomeruli reveal both mesangial and endocapillary hypercellularity with predominant neutrophils (PAS x 200). There is mild tubular atrophy and interstitial fibrosis (involving $25 \%$ of renal cortex); c. direct immunofluorescence microscopy with fluorescein anti-IgA labelling showed mesangial IgA deposit.

teinuria being the most significant prognostic factor. ${ }^{9,10}$ The definitive diagnosis requires a biopsy of the skin and/or kidney. Pathognomonic features include leukocytoclastic vasculitis and IgA deposits in the dermal capillaries and $\lg \mathrm{A}$ deposits in the mesangium."

The use of glucocorticoids is thought to be beneficial for resolving the arthralgias and abdominal pain of HSP. High dose methylprednisolone may be beneficial in patients with advanced renal disease (crescentic nephritis). ${ }^{12}$ Alternative therapies include azathioprine, high-dose IV immunoglobulin, mycophenolate mofetil, cyclophosphamide, and thalidomide. ${ }^{13,14}$ There may be a role for plasmaphoresis in the management of HSP. ${ }^{15}$ However, there have been no randomized clinical trials to document the efficacy of any of these therapeutic regimens.

In summary, adult onset HSP is a rare disease, often missed at initial presentation. An aggressive diagnostic approach should be employed promptly in such patients to make a timely diagnosis to achieve good clinical outcome. Early initiation of high dose steroids may prevent permanent kidney damage in patients like the present case.

Author Affiliation: All authors except Anand Reddy work in the Department of Internal Medicine at Texas Tech University Health Sciences Center in Odessa, TX. Jay Patel and Imran Umer are residents, Yasir Ahmed is an infectious disease specialist, and Craig Spellman is an endocrinologist. Anand Reddy works as clinical nephrologist at TTUHSC in Odessa, TX.

Received: $11 / 10 / 2014$

Accepted: 11/23/2014

Reviewers: Vaqar Ahmed MD

Published electronically: 01/15/2015

Conflict of Interest Disclosures: none 


\section{REFERENCES}

1. Pillebout E, Thervet E, Hill G, Alberti C, Vanhille P, Nochy D. Henoch-Schönlein purpura in adults: Outcome and prognostic factors. J Am Soc Nephrol 2002; 13: 1271-1278.

2. Aktas B, Topcuoglu P, Kurt OK, Ensari A, Demirer T. Severe Henoch- Schönlein purpura induced by cytarabine. Ann Pharmacotherapy 2009; 43(4): 792-793.

3. Zhang Y, Huang X: Gastrointestinal involvement in HenochSchönlein purpura. Scand J Gastroenterol 2008, 43:1038-1043. 4. Nielsen HE. Epidemiology of Schönlein-Henoch purpura. Acta Paediatr Scand 1988; 77: 125-131.

5. Miura M, Nomoto Y, Sakai H, Yamamoto O. An aged patient with Henoch-Schönlein purpura nephritis: A case report and review of the literature. Intern Med 1992; 31: 232-238.

6. Watts RA, Scott D. Epidemiology of the Vasculitides. Semin Resp Crit Care 2004; 25: 455-464.

7. Borrás-Blasco J, Enriquez R, Amoros F, Cabezuelo JB, Navarro-Ruiz A, Pérez M, Fernández J. Henoch- Schönlein purpura associated with clarithromycin. Case report and review of literature. Intern J Clin Pharmacol Thera 2003; 41(5): 213-216.

8. Saulsbury F.T. Henoch- Schönlein purpura in children. Report of 100 patients and review of the literature. Medicine (Baltimore) 1999; 78: 395-409.

9. Kellerman PS. Henoch-Schönlein purpura in adults. Am J Kid Dis 2006; 48: 1009-1016.

10. Coppo R, Andrulli S, Amore A, Gianoglio B, Conti G, Peruzzi L, et al. Predictors of outcome in Henoch- Schönlein nephritis in children and adults. Am J Kid Dis 2006; 47: 993-1003.

11. Kauffmann RH, Herrmann WA, Meÿer CJ, Daha MR, Van Es LA. Circulating IgA-immune complexes in Henoch-Schönlein purpura. A longitudinal study of their relationship to disease activity and vascular deposition of IgA. Am J Med 1980; 69: 859866.

12. Niaudet $P$, Habib R. Methylprednisolone pulse therapy in the treatment of severe forms of Schönlein-Henoch purpura nephritis. Pediatr Nephrol 1998; 12: 238-243.

13. Bergstein J, Leiser J, Andreoli SP. Response of crescentic Henoch- Schönlein purpura nephritis to corticosteroid and azathioprine therapy. Clin Nephrol 1998; 49: 9-14.

14. Dede F, Onec B, Ayli D, Gounul II, Onec K. Mycophenolate mofetil treatment of crescentic Henoch- Schönlein nephritis with IgA depositions. Scand J Urol Nephrol 2008; 42: 178-180.

15. Kauffmann RH, Houwert DA. Plasmapheresis in rapidly progressive Henoch-Schoenlein glomerulonephritis and the effect on circulating IgA immune complexes. Clin Nephrol 1981; 16:155. 